\title{
Anterior abdominal fixation - a new option in the surgical treatment of pelvic organ prolapse
}

\author{
Jakub Sliwa®, Anna Kryza-Ottou, Justyna Grobelak, \\ Zygmunt Domagala, Mariusz Zimmer(B)
}

Departament of Obstetrics and Gynecology, Medical University of Wroclaw, Poland

\begin{abstract}
Objectives: To present anterior abdominal fixation - a new surgical technique for the treatment of pelvic organ prolaps (POP) and to evaluate the results of the treatment of patients with stage III and IV POP operated using this technique.

Material and methods: Anterior abdominal fixation for treating stage III and IV POP was carried out in 42 women, who were qualified according to the Pelvic Organ Prolapse Quantification System (POP-Q) scale at baseline and after 12 months. The Pelvic Floor Disability Index-20 (PFDI-20), along with its symptom scales, were evaluated.

Results: The mean age 42 operated women was 64.5 years, and the average BMI was 27.3 ( $83 \%$ women were overweight). At baseline, 29 (69\%) women had POP stage IV, and 13 (31\%) women had POP stage III. Overall, 14 (33\%) underwent laparoscopy, 28 (67\%) underwent laparotomy. At 12 months, 14 (33.3\%) women had POP stage I; 21 (50\%) women had POP stage II. Seven patients (16.6\%) experienced a recurrence of disease with advancement at the degree of III/IV; 4 (9.5\%) women required adjuvant surgery in the form of anterior and posterior vaginal wall surgery. No early complications after surgery were observed. The comparison of the results before and after surgery showed statistically significant improvement in terms of the P-QoL score as well as PFDI-20 along with its 3 symptom scales.

Conclusions: Anterior abdominal fixation of the uterus to the anterior abdominal wall is effective, safe, and technically easy to perform in the treatment of POP of advanced stage.
\end{abstract}

Key words: pelvic organ prolapse; quality of life; women's health

Ginekologia Polska 2021; 92, 7: 471-474

\section{INTRODUCTION}

Pelvic organ prolapse (POP) in women is defined as the lowering of the pelvic organs, leading to the protrusion of the vagina, cervix or the entire uterus. It mainly affects postmenopausal women and is accompanied by symptoms related to the lowering of the urethra, bladder, small intestine and/or rectum [1]. Risk factors of POP can be divided into congenital factors, which include disorders of the connective tissue structure, abnormal innervation of the pelvic organs, and defects in the build of the pelvis or the spine, as well as acquired factors, which include multiple vaginal births, heavy physical work, a chronic cough, constipation, age, obesity, and the removal of the uterus $[2,3]$. The most common symptoms of POP include: a feeling of heaviness in the vagina, noticeable protrusion of the uterus or vaginal walls through the labia, abnormal urination and/or defecation, sexual dysfunction [4].
In the treatment of pelvic organ prolapse, conservative treatment methods are used first. They include: weight reduction, training of the pelvic diaphragm muscles (Kegel exercises), and also pharmacological improvement of the quality of the vaginal mucosa and vaginal pessaries. In the case of ineffective conservative treatment, surgical treatment of POP, which is performed by transvaginal or transabdominal access, is used. It is conducted using laparotomy or laparoscopy and involves the suspension of the uterus or vagina to the uterosacral ligaments or sacrospinous ligaments, or is performed using Mayo culdoplasty or the McCall method [5].

Despite the use of many surgical techniques in the treatment of POP in women, there is still no single method that is safe, effective and that has a short learning curve. In the past, for the so-called "gold standard" of surgery, some operators recognized sacrocolpopexy and/or sacrouteropexy. This is due to the fact that this operation suspends the vagina and

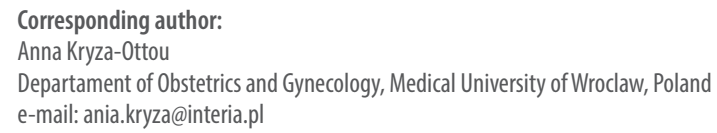


uterus posteriorly in the vaginal axis and has a high degree of effectiveness. On the other hand, because of the possibility of the occurrence of serious complications (damage to the organs and vessels of the smaller pelvis, pre-sacral haemorrhage, defecation disorders, adhesions, urinary incontinence), and also the high degree of difficulty of the procedure - this type of surgery nowadays does not meet the gold standard criterion [6]. When choosing the optimal technique for a given patient, it is important to take into account the current type of defect, the patient's age, physical activity, obstetric interview, procreation plans, operations in this area and, above all, the patient's preferences. A similarly important element in the selection of the optimal surgical technique is the degree of difficulty of the procedure and the operator's experience [5].

In recent years, there has been increased attention paid towards patient's preferences and concerns about the impact on the quality of sexual intercourse, and also the psychological feelings associated with removing or saving the uterus. As a result, there is a tendency to decrease the number of hysterectomies in favour of sparing surgical procedures that preserve the uterus [7-9]. The surgical techniques of POP with the saving of the uterus include, among others, suspension of the uterus to the uterosacral ligaments, sacrouteropexy, pectopexy, and the Manchester technique [6]. Effective surgical treatment of pelvic organ or vaginal stump prolapse requires apical suspension, either with the option of removing the uterus, or saving it. Most of the results of surgical treatment of POP come from retrospective data analyses - the preferred surgical access is undoubtedly vaginal and laparoscopic access as it is minimally invasive and does not require the cutting of the peritoneal cavity [5]. Regarding the above assumptions, in the $2^{\text {nd }}$ Department of Obstetrics and Gynecology of Wroclaw Medical University, Wrocław, Poland, a new surgical technique for the treatment of POP in women in stages III and IV, according to the Pelvic Organ Prolapse Quantification System (POP-Q) scale, was developed. It involves the laparoscopic suspension of the uterus to the anterior abdominal wall - that is, in the anterior direction by means of transfascial insoluble sutures and with the use of a polypropylene mesh applied retroperitoneally and supra-fascially - anterior abdominal fixation.

The aim of the study was to present a new surgical technique for the treatment of POP and to initially evaluate the results of the treatment of patients with stage III and IV POP assessed on the POP-Q scale who were operated on in the $2^{\text {nd }}$ Department of Gynecology and Obstetrics using the technique described below.

\section{MATERIAL AND METHODS}

In this study, the effect of surgical treatment of 42 women operated on due to pelvic organ prolapse, who were in stages III and IV according to the POP-Q scale, was assessed. The patients underwent surgery that involved the suspension of a pelvic organ (uterus, cervix or vaginal stump) to the fascia of the straight abdominal muscles with the use of insoluble monofilament threads, and also the protection of insoluble sutures with a polypropylene mesh that was fixed to the anterior abdominal wall. The effect of the treatment was assessed using the POP-Q scale during a gynecological examination 12 months after the surgery.

Until now, surgical procedures using the surgical method presented in this study have been performed for $42 \mathrm{pa}$ tients with stage III or IV POP according to the POP-Q scale. The method involved the laparoscopic or laparotomy suspension of the uterus in the anterior direction, i.e. to the anterior abdominal wall, with the use of two insoluble monofilament sutures and a polypropylene mesh applied supra-fascially. The mesh was used to strengthen the flaccid fascia of the rectus abdominis, and also to reinforce non-dissolving sutures. Being an artificial material, the mesh was implanted retroperitoneally over the fascia of the rectus abdominis, which makes the procedure easy and effective, and at the same time, excludes the risk of adhesions and erosion of the mesh into the peritoneal cavity. The operation enables the uterus to be saved, which reduces the invasiveness of the method, as well as shortens the procedure time. The use of the described surgical technique was approved by the Bioethics Committee of Wroclaw Medical University, Wrocław, Poland.

\section{The technique of the procedure}

The following steps show how to perform the operation:

1. Placement of the manipulator into the uterine cavity through the cervical canal in order to obtain uterine mobility.

2. Introduction of standard laparoscopic equipment to gain access to the abdominal cavity. One optical trocar and two side trocars are used.

3. Approximately $3 \mathrm{~cm}$ above the pubic symphysis, a $3 \mathrm{~cm}$ long transverse skin incision is made and the subcutaneous tissue is bluntly separated to the level of the rectus abdominus fascia; the fascia is not incised.

4. The first retroperitoneal suture for the uterus - the first suture is made with a straight needle and involves the puncturing of the fascia, rectus abdominis, and peritoneum into the peritoneal cavity. The needle is then punctured through the uterus at a specific point, i.e. at the level of the isthmus, above the entrance of the uterine arteries - from the front of the uterus to the back - and then in the opposite direction - from the posterior wall of the uterus to the front at the same level - and again through the muscles and fascia to the outside (U-shaped suture). The determination and 
precise puncture in the marked place, i.e. at the level of the isthmus and above the entrance of the uterine arteries, is critical as it provides a safe distance from the uterine artery and a low risk of complications.

5. The second transperitoneal suture - a straight needle is also used, and the steps are the same as for the first suture - the uterus is punctured slightly above the first suture.

6. Fixing the mesh directly over the fascia - the mesh used in the procedure has dimensions of $4 \times 2 \mathrm{~cm}$. The mesh is placed under the subcutaneous tissue, exactly in the place of the previously prepared fascia. The sutures that were conducted through the uterus and mesh to the abdominal wall, when the mobile uterus is pulled up to the abdominal wall, they are tied together over the mesh.

7. When the sutures are tied, the uterus is pulled up and forward towards the abdominal wall in such a way that its anterior wall meets the peritoneum of the anterior boundaries of the abdominal cavity.

Data concerning demographic information, disease advancement on the POP-Q scale and the type and course of surgery in 42 operated women were evaluated at baseline. Additionally, the subjective severity of pelvic floor complaints was assessed on the basis of the Pelvic Organ Prolapse Quality of Life (P-QoL) questionnaire and the Pelvic Floor Disability Index-20 (PFDI-20) questionnaire. The data from the PFDI-20 questionnaire were divided into groups of questions concerning symptoms associated with POP using the Pelvic Organ Prolapse Distress Inventory 6 (POPDI-6), symptoms associated with distal gastrointestinal disorders using the Colorectal-Anal Distress Inventory 8 (CRAD-8), and symptoms associated with the dysfunction of the urinary system using the Urinary Distress Inventory 6 (UDI-6). After 12 months, the results of the procedures were assessed by re-examining the advancement of the disease and perceived symptoms.

The collected data were statistically analysed. The data were presented as means and standard deviations as well as numbers and percentages. The Kolmogorov-Smirnov test was used to test for normal distribution. The Wilcoxon signed-rank test both for paired comparisons was used. Data were considered to be statistically significant at a value of $p<0.05$. Statistical analysis was carried out with Statistica software (StatSoft, Tulsa, OK, USA).

\section{RESULTS}

The average age of the operated women was 64.5 years, the average weight was $71 \mathrm{~kg}$, the average height was $1.62 \mathrm{~m}$, and the average BMI was 27.3 (83\% were overweight patients). As many as $69 \%$ of the patients reported being involved in physical work in the past or now, and the re- maining 31\% performed white-collar work, which confirms the influence of physical work on the degree of the defect.

The operated patients reported on average 2 vaginal childbirth deliveries. The average weight of a newborn was $3651 \mathrm{~g}$, with a minimum of 2650 grams and a maximum of $4500 \mathrm{~g}$. Such a high weight had at least $25 \%$ of the newborns. In the preoperative examination, the disease was assessed as stage IV according to POP-Q in 29 women (69\%), and as stage III in 13 women (31\%).

Overall, 14 (33\%) operations were performed using laparoscopy, and the remaining 28 (67\%) using laparotomy. In 14 (33\%) patients, conservative uterine-sparing surgery was performed. The average time of the procedure, including the preparation of the patient, was 115 minutes in the case of laparotomy, and 85 minutes in the case of laparoscopy. The mean blood loss during the procedure, assessed on the basis of the decrease in the haemoglobin level in the venous blood count, was $1.66 \mathrm{mg} / \mathrm{dL}$, with no significant differences in both groups.

In 35 out of 42 patients (83.3\%), clinical improvement was observed. A gynecological postoperative examination 12 months after the surgery revealed that the degree of organ prolapse on the POP-Q scale was on average I/II. In details, 14 (33.3\%) women had POP stage I, and 21 (50\%) patients had POP stage II. Seven (16.6\%) patients had a recurrence of disease with an advancement at stage III/IV on the POP-Q scale 12 months after the surgery. Four (9.5\%) patients required adjuvant surgery in the form of anterior and posterior vaginal wall surgery.

No early complications after surgery were observed in any of the patients.

At baseline and 12 months after surgery, the subjective feelings related to pelvic floor problems in the operated patients were assessed on the basis of self-completed P-QoL and PFDI-20 questionnaires and both showed significant improvement. Based on these scales, 35 (83.3\%) women had a significant subjective improvement in perceived ailments related to POP, as well as an improvement in the quality of life. The postoperative results of 7 (16.6\%) women were comparable to those before the operation.

The comparison of the results before and after surgery showed statistically significant differences between the studied P-QoL domains. Also the change in the score from baseline to at 12 months showed significant improvement for all questionnaires (PFDI-20, POPDI-6, CRAD-8, UDI-6) which scores are presented in Table 1 . The average level of PFDI-20 before the surgery is more than twice as high as after the surgery, which means a significant improvement in the perception of pelvic floor problems. The frequency of experiencing the examined symptoms and their impact on life after the surgery is statistically significantly different from the frequency before the surgery. 
Table 1. Results of the assessment of pelvic organ prolapse before and after the surgery

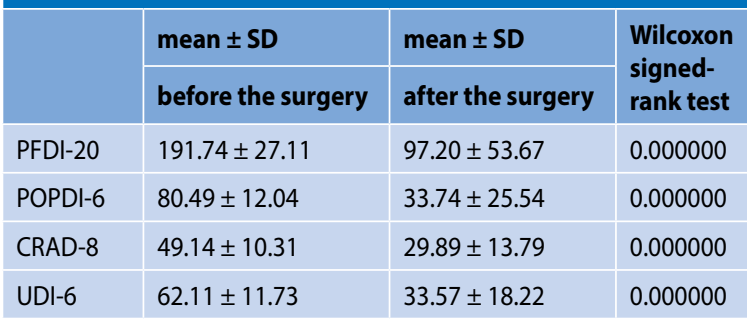

CRAD-8 - Colorectal-Anal Distress Inventory 8; PFDI-20 — Pelvic Floor Disability Index-20; POPDI-6 — Pelvic Organ Prolapse Distress Inventory 6 SD - standard deviation; UDI-6 - Urinary Distress Inventory 6

After the surgery, subjective and objective improvement was observed in most of the operated patients with regards to vagina and uterine prolapse, as well as to the urinary system. This was especially the case for those with advanced POP and urine retention before the surgery. It should be emphasized at this point that the discomfort of patients with POP stage III/IV is so great that some imperfections of the method, such as pulling the pelvic organ in the anterior direction in the non-physiological vaginal axis, or the need to execute additional vaginal wall plastic surgery, are fully acceptable for the patients.

\section{CONCLUSIONS}

The anterior abdominal fixation of the uterus to the anterior abdominal wall is effective, safe, and technically easy to perform in the treatment of POP of advanced stage. It is associated with a lower rate of intraoperative complications than other methods used in POP, e.g. sarcocolpopexy, and a lower risk of damage to large blood vessels. Moreover, the results are satisfying for patients. The technique of uterine suspension using laparoscopy is particularly preferred.
In some patients with a mixed type of POP, it may be necessary to perform additional plastic surgeries of the posterior vaginal wall. The use of a supra-fascial mesh strengthens the flaccid fascia and prevents against the risk of mesh erosion and the development of intraperitoneal adhesions.

\section{Conflict of interest}

The authors declare no conflict of interest.

\section{REFERENCES}

1. Haylen BT, Maher CF, Barber MD, et al. An International Urogynecological Association (IUGA) / International Continence Society (ICS) joint report on the terminology for female pelvic organ prolapse (POP). Int Urogynecol J. 2016; 27(2): 165-194, doi: 10.1007/s00192-015-2932-1, indexed in Pubmed: 26755051.

2. Chow D, Rodríguez LV. Epidemiology and prevalence of pelvic organ prolapse. Curr Opin Urol. 2013; 23(4): 293-298, doi: 10.1097/MOU.0b013e3283619ed0, indexed in Pubmed: 23619578.

3. Śliwa J, Rosner-Tenerowicz A, Kryza-Ottou A, et al. Analysis of prevalence of selected anamnestic factors among women with pelvic organ prolapse. Adv Clin Exp Med. 2018; 27(2): 179-184, doi: 10.17219/acem/68994, indexed in Pubmed: 29521060.

4. Manonai J, Mouritsen L, Palma P, et al. The inter-system association between the simplified pelvic organ prolapse quantification system (S-POP) and the standard pelvic organ prolapse quantification system (POPQ) in describing pelvic organ prolapse. Int Urogynecol J. 2011; 22(3): 347-352, doi: 10.1007/s00192-010-1286-y, indexed in Pubmed: 20936258.

5. Park AJ, Paraiso MFR. Surgical management of uterine prolapse. Minerva Ginecol. 2008; 60(6): 493-507, indexed in Pubmed: 18981977.

6. Kale A, Biler A, Terzi H, et al. Laparoscopic pectopexy: initial experience of single center with a new technique for apical prolapse surgery. Int Braz J Urol. 2017; 43(5): 903-909, doi: 10.1590/S1677-5538.IBJU.2017.0070, indexed in Pubmed: 28727377.

7. Husby KR, Lose G, Klarskov N. Trends in apical prolapse surgery between 2010 and 2016 in Denmark. Int Urogynecol J. 2020; 31(2): 321-327, doi: 10.1007/s00192-018-3852-7, indexed in Pubmed: 30610266.

8. Anglim B, O'Sullivan O, O'Reilly B. How do patients and surgeons decide on uterine preservation or hysterectomy in apical prolapse? Int Urogynecol J. 2018; 29(8): 1075-1079, doi: 10.1007/s00192-018-3685-4, indexed in Pubmed: 29947819.

9. Śliwa J, Kryza-Ottou A, Zimmer-Stelmach A, et al. A new technique of laparoscopic fixation of the uterus to the anterior abdominal wall with the use of overfascial mesh in the treatment of pelvic organ prolapse. Int Urogynecol J. 2020; 31(10): 2165-2167, doi: 10.1007/s00192-02004287-4, indexed in Pubmed: 32303776. 